\title{
Comportamento de Sementes de Peltophorum dubium [(Spreng.) Taub.] em Banco de Sementes Induzido
}

\author{
Girlânio Holanda da Silva ${ }^{1}$, Anderson Cleiton Josée ${ }^{2}$ José Márcio Rocha Faria ${ }^{2}$ \\ ${ }^{1}$ Departamento de Ciência Florestal, Faculdade de Ciências Agronômicas - FCA, Universidade Estadual Paulista \\ "Júlio de Mesquita Filho" - UNESP, Botucatu/SP, Brasil \\ ${ }^{2}$ Departamento de Ciências Florestais, Universidade Federal de Lavras - UFLA, Lavras/MG, Brasil
}

\begin{abstract}
RESUMO
A capacidade que determinadas espécies têm de manter as sementes viáveis por longos períodos no banco de sementes é essencial para a resiliência ambiental, o que é utilizado na restauração florestal. Este trabalho teve como objetivo estudar o comportamento de banco de sementes induzido de $P$. dubium. A indução do banco de sementes foi feita em fragmento de Floresta Estacional Semidecídual. Foram utilizadas 100 amostras de copos de polietileno, compostas por 25 sementes cada. A cada dois meses, 5 amostras foram retiradas para determinação de: conteúdo de água, viabilidade, porcentagem de emergência de plântulas e porcentagem de predação. As sementes da espécie $P$. dubium podem permanecer por até 16 meses viáveis enterradas no solo. Esse resultado permite a classificação da espécie como formadora de banco de sementes persistente. A predação de sementes foi o principal fator para que ocorresse a redução do banco de sementes do solo.
\end{abstract}

Palavras-chave: dormência, viabilidade, longevidade.

\section{Behavior of Peltophorum dubium ((Spreng.) Taub.) Seeds in a Burial Seed Bank}

\begin{abstract}
The ability of certain species in keeping the seeds viable for long periods in the soil seed bank is essential for environmental resilience, which is used in forest restoration. This work aimed to study the behavior of a buried seed bank of P. dubium. Burial seed bank was performed in a fragment of Seasonal Semideciduous Forest. One hundred samples consisting of 25 seeds in polyethylene cups were placed under the canopy. Every two months, five samples were taken for determination of water content, viability, emergency (root protrusion and seedling formation) and predation. Seeds of $P$. dubium may remain buried in the ground for up to 16 months keeping their viability. This result indicates that $P$. dubium is able to form persistent soil seed bank. The predation was the main factor contributing to the seed bank reduction.
\end{abstract}

Keywords: dormancy, viability, longevity. 


\section{INTRODUÇÃO}

A dispersão de sementes e processos associados à germinação, recrutamento e estabelecimento de plântulas, bem como a sobrevivência após o estabelecimento são fatores importantes que modulam a dinâmica de regeneração florestal (Dalling et al., 2002; Almeida \& Galetti, 2007; Mendoza-Hernandez et al., 2013; Chen et al., 2014).

A estratégia de regeneração florestal mais importante é a formação do banco de sementes do solo, o qual é definido como o conjunto de sementes viáveis em determinada área do solo ou misturada a serapilheira em certo período de tempo (Daïnou et al., 2011; Li et al., 2012).

Apesar da importância ecológica do banco de sementes, pouca atenção tem sido dada a esse tema em ecossistemas tropicais. Dessa forma, estudos voltados ao tema podem justificar-se, visto que o banco de sementes é uma fonte significativa para a regeneração florestal e, devido a essa importância, o mesmo tem sido utilizado como alternativa para diminuir o tempo de recuperação de áreas que sofreram algum tipo de distúrbio (Hosogi \& Kameyama, 2006; Williams et al., 2008; O’Donnell et al., 2016).

De acordo com o período de permanência do banco de sementes no solo, o mesmo pode ser classificado em cinco tipos: transitórios, quando suas sementes ficam viáveis no solo por até um ano (germinam rápido quando em condições adequadas); persistentes, quando a viabilidade das sementes no solo ultrapassa um ano; pseudo-persistentes, com sementes de curta longevidade, quando a dispersão ocorre mais de uma vez ao ano; transitório-estacional, quando apresentam sementes de curta longevidade e com dormência em certo período do ano; e transitório-atrasado, composto por sementes cuja germinação é amplamente distribuída no tempo (Garwood, 1989).

A formação do banco de sementes do solo é uma estratégia de regeneração que várias espécies desenvolveram, a qual permite a essas plantas passar por adversidades ambientais ao longo do tempo, de forma a diminuir o risco de extinção. Segundo Baskin \& Baskin (2014), existem mais de 1.300 espécies formadoras de banco de sementes distribuídas em cerca de 160 famílias, algumas das quais podem manter sua viabilidade por décadas (Murdoch \& Ellis, 2000). Além do mais, alguns estudos sugerem que o mesmo mecanismo pode ajudar na manutenção da diversidade genética da população e na ocupação de hábitats que sofreram algum tipo de distúrbio (Fenner \& Thompson, 2005; Mandák \& Placková, 2009; Li et al., 2012).

Sabendo da importância ecológica do banco de sementes, alguns estudos têm focado em compreender a ecofisiologia de bancos de sementes, tendo em vista que tais conhecimentos podem auxiliar no desenvolvimento de programas de recuperação de áreas degradadas (Coffey \& Kirkman, 2006; Kaeser \& Kirkman, 2012).

A espécie $P$. dubium é uma Fabaceae arbórea conhecida como canafístula, encontrada em Florestas Estacionais Semideciduais. Sua madeira é comumente utilizada na construção civil. Por ser de rápido crescimento, é utilizada em projetos de reflorestamento (Lorenzi, 2002; Marchiori \& Alves, 2014); as suas sementes, quando maduras, apresentam forma oblonga, com 0,9-1,2 cm de comprimento e 0,4-0,6 cm de largura e coloração castanho-esverdeada-clara; o peso de mil sementes aproxima-se de 49 gramas (Brüning et al., 2011).

Dessa forma, objetivou-se avaliar o comportamento das sementes de P. dubium em banco de sementes induzido.

\section{MATERIAL E MÉTODOS}

\section{1. Área de estudo}

As análises do banco de sementes induzido foram conduzidas no município de Lavras, MG, em área experimental localizada no campus da Universidade Federal de Lavras (UFLA), nas coordenadas S $-21^{\circ} 13^{\prime} 45^{\prime \prime}$ e W $-44^{\circ} 58^{\prime} 97^{\prime \prime}$ e no Laboratório de Sementes Florestais (LSF) do Departamento de Ciências Florestais da UFLA, entre abril de 2014 e novembro de 2015.

\subsection{Seleção das matrizes e obtenção das sementes}

Para o estudo do banco de sementes induzido, as sementes foram obtidas de quatro matrizes no município, as quais foram encontradas em uma propriedade particular na zona rural do município de Lavras, MG, nas coordenadas $21^{\circ} 16^{\prime} 05^{\prime \prime} \mathrm{S}$ e $45^{\circ} 1^{\prime} 33^{\prime \prime} \mathrm{W}$.

Os critérios de seleção das matrizes foram: uniformidade de maturação dos frutos, verificada pela coloração dos frutos (marrom escuro) e início da 
dispersão natural dos mesmos (Aquino et al., 2006), ausência de frutos da safra anterior e abundância de frutos, distância mínima entre as matrizes de 50 metros e aspecto fitossanitário (ausência de fungos e insetos).

Após a coleta dos frutos, foi imediatamente realizado o beneficiamento dos mesmos, para a instalação do banco de sementes induzido, que se deu no mês de julho de 2014. Os frutos foram beneficiados manualmente, extraindo-se as sementes dos frutos e utilizando-as na simulação do banco de sementes.

\subsection{Instalação do banco de sementes induzido}

Foi instalado um experimento para simular um banco de sementes de P. dubium no solo em um fragmento florestal no campus da UFLA caracterizado como Floresta Estacional Semidecidual, de forma que a quantidade de amostras instaladas fosse suficiente para 10 coletas com periodicidade bimestral.

Para indução do banco de sementes do solo as sementes foram colocadas em copos de polietileno branco (diâmetro inferior de $4,6 \mathrm{~cm}$, diâmetro superior de $7 \mathrm{~cm} \mathrm{e} \mathrm{8,3} \mathrm{cm} \mathrm{de} \mathrm{altura)} \mathrm{com} \mathrm{capacidade} \mathrm{de} 200 \mathrm{ml}$. Os copos foram perfurados no fundo e laterais com agulha de $\cong 0,25 \mathrm{~mm}$ de espessura e preenchidos até dois terços com solo do local. Logo após, foram enterrados até o nível interno do substrato. Os copos foram distribuídos aleatoriamente em grupos de 20 amostras em uma área de $100 \mathrm{~m}^{2}$, em local próximo do centro do fragmento florestal. Em cada copo foram colocadas 25 sementes cobertas com aproximadamente $1 \mathrm{~cm}$ de uma mistura de solo e serrapilheira, seguindo metodologia adaptada proposta por Motta et al. (2006).

As coletas de cinco amostras (copos contendo 25 sementes) do banco de sementes induzido se deram periodicamente, a cada 60 dias. Essas foram levadas para o LSF/UFLA para as seguintes análises: quantificação de matéria seca de sementes, do conteúdo de água, verificação de viabilidade, de predação de sementes e de emergência no banco de sementes (protrusão de radícula e formação de plântulas).

\subsection{Análise da emergência de plântulas}

Para determinação da emergência, a avaliação foi realizada bimestralmente, através da contagem do número de sementes com protrusão radicular e plântulas em cada amostra coletada em campo.

\subsection{Sementes predadas}

A determinação do número de sementes predadas foi realizada de acordo com metodologia adaptada de Motta et al. (2006), fazendo-se a contagem das sementes intactas (SI), sementes germinadas e emergência de plântulas (NP), e fazendo-se a subtração das sementes desaparecidas durante o período para se obter o número de sementes predadas (SP); conforme representada pela Fórmula 1 a seguir:

$S P=25-(S I-N P)$

em que: $\mathrm{SP}=$ número de sementes predadas na amostra; $\mathrm{SI}=$ número de sementes intactas, $\mathrm{NP}=$ número de plântulas.

\subsection{Viabilidade}

Para esse teste, as sementes intactas restantes nas amostras foram colocadas para germinar, a cada dois meses, em laboratório.

A viabilidade das sementes de $P$. dubium foi determinada pela germinação, utilizando-se como substrato rolo de papel com 4 repetições com as sementes restantes das amostras que inicialmente continham 25 sementes. Antes do teste de germinação foi realizada superação da dormência física nas sementes realizando-se um corte de aproximadamente $1 \mathrm{~mm}$ no tegumento, no lado oposto da micrópila, utilizando-se uma lâmina. Após a superação da dormência, foi realizada a desinfestação das sementes em solução de hipoclorito de sódio 1\% durante três minutos, seguida de lavagem em água destilada por um minuto. O experimento foi conduzido em câmara de germinação a $25^{\circ} \mathrm{C}$, com fotoperíodo de 12 horas, e avaliado diariamente até o sétimo dia. Como critério de germinação foi considerada a protrusão da radícula ( $\geq 1,0 \mathrm{~mm})$.

\subsection{Conteúdo de água}

O conteúdo de água das sementes foi determinado pelo método da estufa, como recomendado por Brasil (2009). Em cada período de análise foi retirada uma amostra contendo inicialmente 25 sementes. Após limpeza superficial, foi realizada a preparação das amostras, dividindo-se as sementes em 4 repetições de pelo menos $0,3 \mathrm{~g}$, pesadas em balança analítica $(0,0001 \mathrm{~g})$, com a finalidade da aferição da matéria fresca; em seguida as sementes foram incubadas em estufa a 
$105^{\circ} \mathrm{C}$ por 24 oras e logo após novamente pesadas, para a obtenção da matéria seca. Como as sementes apresentavam tegumento rígido, antes da determinação do conteúdo de água foram cortadas ao meio.

\subsection{Determinação da umidade de solo}

Amostras de solo foram analisadas bimestralmente, fazendo-se a amostragem no momento da retirada das sementes para as análises fisiológicas. Foram coletadas aleatoriamente quatro amostras do solo localizado no entorno do banco de sementes induzido. A determinação da umidade do solo foi realizada seguindo-se a metodologia proposta pela Empresa Brasileira de Pesquisa Agropecuária (Embrapa, 1997).

\subsection{Dados climatológicos}

Os dados de precipitação foram obtidos na estação climatológica da UFLA, situada a cerca de um quilômetro do local do experimento.

\subsection{Delineamento experimental e análises estatísticas}

O experimento do banco de sementes induzido foi montado em delineamento inteiramente casualizado (DIC), com nove épocas de coletas e quatro repetições. Após tabulação, os dados percentuais de germinação, emergência, predação e número de sementes mortas foram submetidos ao teste de normalidade de Shapiro-Wilk; os dados de viabilidade de sementes foram transformados para $\log (\mathrm{x})$. Após verificação da normalidade dos dados, realizou-se a análise de variância e as médias foram submetidas à análise de regressão. $\mathrm{O}$ modelo de regressão foi determinado baseando-se na significância dos coeficientes da equação de regressão e de determinação a $5 \%$ de probabilidade.

\section{RESULTADOS}

Após a indução do banco de sementes, em julho, pode-se observar variações no conteúdo de água das sementes durante todo o período do estudo. As maiores variações observadas ocorreram entre os meses de julho e setembro de 2015, quando o conteúdo de água variou de $11,6 \%$ para 7,7\% (Figura 1). Observa-se que apesar de pequenas variações no conteúdo de água

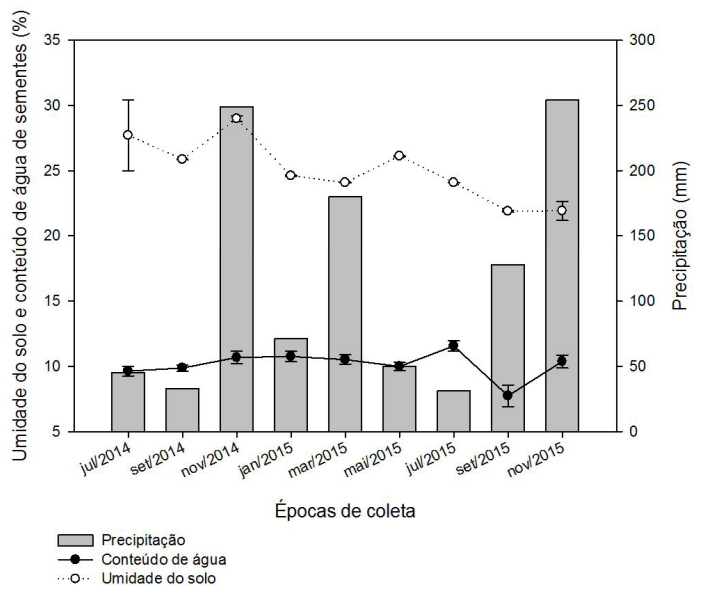

Figura 1. Precipitação mensal, umidade do solo e conteúdo de água das sementes de $P$. dubium no período de julho de 2014 a novembro de 2015. As barras no conteúdo de água e umidade do solo representam o desvio padrão da média.

Figure 1. Monthly rainfall, soil moisture and water content of $P$. dubium seeds from july 2014 to november 2015. The bars on the water content and soil moisture represent the mean standard deviation.

terem ocorrido durante o período de estudo, essa variação não está associada com a precipitação mensal observada, visto que em meses de alta precipitação o conteúdo de água das sementes foi próximo ao dos meses de baixa precipitação. Para a umidade do solo, os valores registrados variaram de $28 \%$, em novembro de 2014 , a $22 \%$, em novembro de 2015.

De acordo com a análise de variância ( $\mathrm{p} \leq 0,05)$, o período de permanência das sementes no banco induzido influenciou de forma significativa a emergência de plântulas de $P$. dubium, entretanto o coeficiente de determinação $\left(\mathrm{R}^{2}\right)$ foi de $11,02 \%$. De maneira geral, a emergência foi alta nos meses de maior precipitação e reduzida no período de seca.

Foi verificado efeito do tempo sobre a viabilidade e predação das sementes no banco de sementes induzido pela análise de variância. Os valores máximos e mínimos da viabilidade de sementes foram observados na primeira e última análises feitas, respectivamente; contrariamente, para o número de sementes predadas no banco, os menores valores foram obtidos no início de experimento e os máximos, ao final (Figura 2).

Durante o período de estudo do banco de sementes do solo verificou-se que a viabilidade decresceu de forma lenta, permanecendo acima de 50\% na maioria 
dos meses no período de julho de 2014, com $84 \%$ de germinação, a setembro de 2015 , com $58 \%$ de germinação, entretanto houve uma queda abrupta na viabilidade em novembro de 2015 (Figura 3).

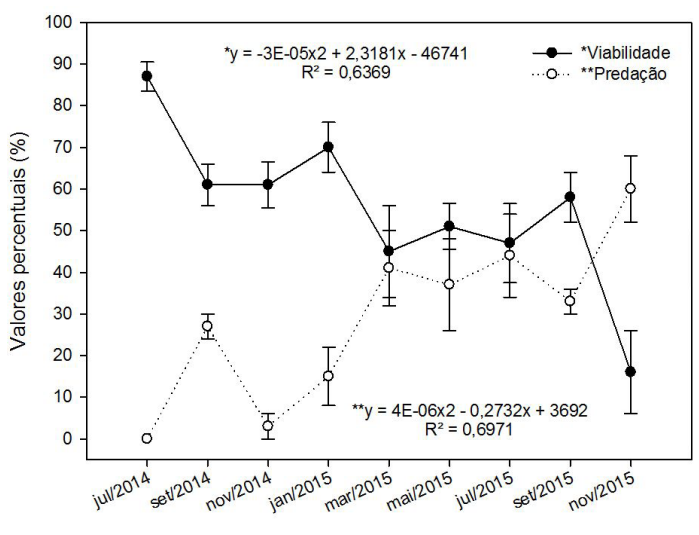

Épocas de coleta

Figura 2. Valores observados e modelos ajustados para estimativa da viabilidade e predação em sementes de P. dubium em banco de sementes induzido. As barras no conteúdo de água e umidade do solo representam o desvio padrão da média.

Figure 2. Observed values and adjusted models to estimate the viability and predation on $P$. dubium seeds in burial seed bank. The bars on the water content and soil moisture represent the mean standard deviation.

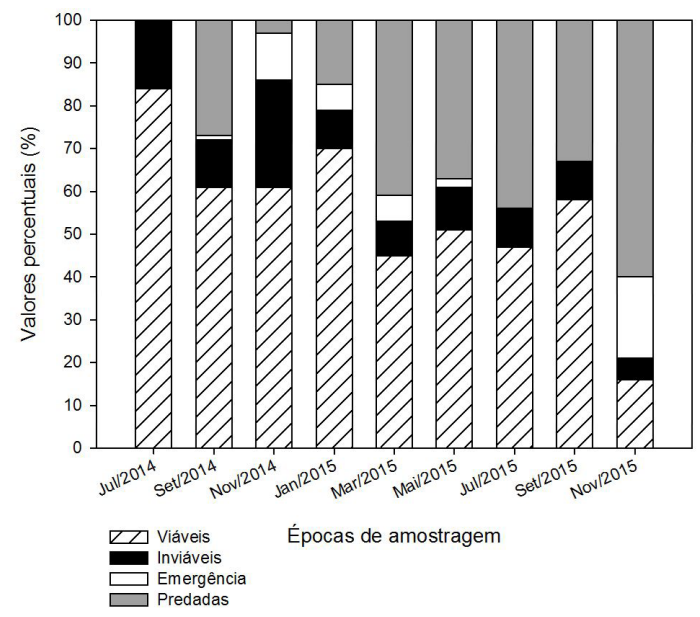

Figura 3. Porcentagem de sementes viáveis, inviáveis (sementes mortas), com emergência de plântulas e sementes predadas em banco de sementes de $P$. dubium induzido no solo, no período de julho de 2014 a novembro de 2015.

Figure 3. Percentage of viable seeds, nonviable (dead seeds), seedling emergence and preyed seeds of $P$. dubium seeds induced in the burial seeds bank, from july 2014 to november 2015 .
A emergência de plântulas ao longo dos períodos analisados foi altamente variável: pôde ser observado que os meses que proporcionaram melhores condições para emergência de plântulas foram os de novembro a março, com destaque para o mês de novembro, com taxas de $11 \%$ e $19 \%$ de plântulas formadas para os anos de 2014 e 2015, respectivamente (Figura 3). Por outro lado, de forma geral, os meses de julho e setembro foram os meses em que a taxa de emergência de plântulas foram menores, com $0 \%$ de emergência.

A taxa de predação ao longo dos períodos analisados foi crescente, de forma que os valores de predação de sementes variaram de $3 \%$ a $60 \%$, correspondendo ao meses de novembro de 2014 e 2015, respectivamente (Figura 3). Observa-se uma tendência de aumento da predação em relação ao período em que as sementes permanecem no banco de sementes do solo.

\section{DISCUSSÃO}

O percentual de água do solo variou de forma significativa entre as coletas feitas entre os anos de 2014 e 2015 (Figura 1). Contudo, essas diferenças parecem não ter afetado o conteúdo de água das sementes. Isso denota a presença de dormência física para a entrada de água na semente (Aquino et al., 2006; Baskin \& Baskin, 2014).

Em algumas espécies que formam banco de sementes, o conteúdo de água é alterado em decorrência de oscilações da umidade do solo, o que pode contribuir para liberação da dormência e, consequentemente, aumento na germinação (Long et al., 2012), porém não há indicação de que as oscilações da umidade do solo e conteúdo de água das sementes influenciem a germinação das sementes (Figuras 1 e 3).

Banco de sementes do solo é definido como a reserva viável de um conjunto de sementes que se encontra no solo ou misturado a serapilheira em determinada área. O banco de sementes pode ser classificado em várias categorias (Garwood, 1989; Baskin \& Baskin, 2014). Dentro do sistema de classificação utilizado para espécies tropicais proposto por Garwood (1989), a espécie $P$. dubium é enquadrada no tipo de banco chamado de persistente, visto que foi verificada a presença de sementes viáveis por até 16 meses, com viabilidade superior a 50\% nos primeiros 14 meses de estudo. 
Uma das possíveis causas que promoveram a longevidade da espécie em estudo foi a presença de tegumento espesso, como evidenciado pela sua dormência tegumentar, o que pode limitar a ação de agentes associados à deterioração, especialmente microoganismos. De acordo com alguns autores, a dormência tegumentar é um dos fatores que contribuem para o aumento da longevidade das sementes (Long et al., 2012, 2015; Hanin et al., 2013; Jha et al., 2015).

Um dos fatores que contribui para a manutenção da viabilidade das sementes ortodoxas é a manutenção do baixo conteúdo de água e armazenamento em ambientes com baixa temperatura (Kanazawa et al., 2015; Suriyong et al., 2015). Neste estudo, apesar da grande variação da temperatura durante o ano, o conteúdo de água das sementes se manteve em torno de $10 \%$ durante todo o experimento, o que pode ter propiciado condições adequadas para a manutenção da viabilidade nas condições de campo (Motta et al., 2006; Shen et al., 2011).

Diversos são os fatores que podem afetar a persistência do banco de sementes do solo, dentre eles os mais significativos são os climáticos e biológicos como temperatura, umidade do solo e incidência de insetos e patógenos (Iglesias-Fernández et al., 2011; Sánchez-Coronado et al., 2011; Long et al., 2015). Nesse contexto, diversos trabalhos relatam que o nível de predação no banco de sementes é a principal causa da persistência do mesmo (Bruun et al., 2010).

Também pôde ser observado no presente trabalho aumento na predação das sementes durante o período do estudo. Nos primeiros seis meses após a indução do banco de sementes observou-se uma predação de aproximadamente $11 \%$ das sementes. Após esse período, a predação aumentou até atingir $60 \%$, na última análise, além disso pôde ser notado que a taxa no aumento da predação foi semelhante ao padrão da taxa de queda na viabilidade de sementes ao longo do tempo. Segundo Van Klinken \& White (2014), oscilações de baixa e alta predação de sementes no solo no início do banco de sementes é bastante comum em florestas tropicais.

A predação de sementes é bastante relacionada com a profundidade em que as sementes se encontram no solo, de modo que sementes em maiores profundidades são menos susceptíveis a predação, mas, por outro lado, a profundidade pode também diminuir a emergência de plântulas (Delefosse \& Kristensen, 2012; Jurand et al.,
2013); neste trabalho a taxa de predação aumentou de forma significativa ao longo do tempo considerando apenas a profundidade $1 \mathrm{~cm}$.

O padrão intermitente da emergência de plântulas de $P$. dubium somente em alguns períodos do ano sugere que a espécie possui estratégia de distribuição temporal da germinação, o que é comum em espécies formadoras de banco de sementes do solo (Hong et al., 2012; Baskin \& Baskin, 2014). Essa estratégia reduz o risco de extinção, seja por predação ou condições climáticas (Brits et al., 2015). Além disso, o baixo índice de emergência de plântulas da espécie $P$. dubium em julho e setembro sugere alta pressão do ambiente durante o desenvolvimento vegetal nesses meses que correspondem ao inverno na região, o que pode ser causado pela baixa temperatura e baixa precipitação ocorridas (Figura 1) (Mendoza-Hernández et al., 2014; Benítez-Rodríguez et al., 2014; Soriano et al., 2014; Limón \& Peco, 2016).

\section{CONCLUSÕES}

Sementes de P. dubium formam banco de sementes persistente, mantendo viabilidade acima de $50 \%$ por 14 meses.

A predação foi o principal fator que contribuiu para a redução do banco de sementes do solo.

\section{STATUS DA SUBMISSÃO}

Recebido: 2 jun., 2016

Aceito: 20 nov., 2016

\section{AUTOR(ES) PARA CORRESPONDÊNCIA}

\section{Girlânio Holanda da Silva}

Departamento de Ciência Florestal, Faculdade de Ciências Agronômicas - FCA, Universidade Estadual Paulista "Júlio de Mesquita Filho" UNESP, Rua José Barbosa de Barros, $n^{\circ}$ 1780, CEP 18610-307, Botucatu, SP, Brasil e-mail: girlanio_holanda@hotmail.com

\section{REFERÊNCIAS}

Almeida LB, Galetti M. Seed dispersal and spatial distribution of Attalea geraensis (Arecaceae) in two remnants of Cerrado 
in Southeastern Brazil. Acta Oecologica 2007; 32(2): 180-187. http://dx.doi.org/10.1016/j.actao.2007.04.001.

Aquino NF, Bortolini M, Campagnolo MA, Ignácio VL, Kopper AC, Malavasi MM. Dormência de sementes de Peltophorum dubium (Sprengel.) Taubert colhidas em diferentes estádios de desenvolvimento. Scientia Agraria Paranaensis 2006; 5(2): 31-37.

Baskin CC, Baskin JM. Seeds: ecology, biogeography, and evolution of dormancy and germination. 2nd. ed. San Diego: Academic: Elsevier; 2014.

Benítez-Rodríguez L, Gamboa-de Buen A, SánchezCoronado ME, Alvarado-López S, Soriano D, Méndez I et al. Effects of seed burial on germination, protein mobilisation and seedling survival in Dodonaea viscosa. Plant Biology 2014; 16(4): 732-739. PMid:24148161. http:// dx.doi.org/10.1111/plb.12110.

Brasil. Ministério da Agricultura, Pecuária e Abastecimento. Regras para análise de sementes. Brasília: Secretaria de Defesa Agropecuária; 2009.

Brits GJ, Brown NAC, Calitz FJ, Van Staden J. Effects of storage under low temperature, room temperature and in the soil on viability and vigour of Leucospermum cordifolium (Proteaceae) seeds. South African Journal of Botany 2015; 97: 1-8. http://dx.doi.org/10.1016/j.sajb.2014.11.003.

Brüning OF, Lúcio ADC, Muniz MFB. Padrões para germinação, pureza, umidade e peso de mil sementes em análises de sementes de espécies florestais nativas do Rio Grande do Sul. Ciência Florestal 2011; 21(2): 193-202. http://dx.doi.org/10.5902/198050983221.

Bruun HH, Valtinat K, Kollmann J, Brunet J. Postdispersal seed predation of woody forest species limits recolonization of forest plantations on ex-arable land. Preslia 2010; 82: 345-356.

Chen L, Wang L, Baiketuerhan Y, Zhang C, Zhao X, von Gadow K. Seed dispersal and seedling recruitment of trees at different successional stages in a temperate forest in northeastern China. Journal of Plant Ecology 2014; 7(4): 337-346. http://dx.doi.org/10.1093/jpe/rtt024.

Coffey KL, Kirkman LK. Seed germination strategies of species with restoration potential in a fire-maintained pine savanna. Natural Areas Journal 2006; 26(3): 289-299. http://dx.doi.org/10.3375/0885-8608(2006)26[289:SGS OSW]2.0.CO;2.

Daïnou K, Bauduin A, Bourland N, Gillet JF, Fétéké F, Doucet JL. Soil seed bank characteristics in Cameroonian rainforests and implications for post-logging forest recovery. Ecological Engineering 2011; 37(10): 1499-1506. http:// dx.doi.org/10.1016/j.ecoleng.2011.05.004.

Dalling JW, Muller-Landau HC, Wright SJ, Hubbell SP. Role of dispersal in the recruitment limitation of neotropical pioneer species. Journal of Ecology 2002; 90(4): 714-727. http://dx.doi.org/10.1046/j.1365-2745.2002.00706.x.
Delefosse M, Kristensen E. Burial of Zostera marina seeds in sediment inhabited by three polychaetes: laboratory and field studies. Journal of Sea Research 2012; 71: 41-49. http://dx.doi.org/10.1016/j.seares.2012.04.006.

Empresa Brasileira de Pesquisa Agropecuária - EMBRAPA. Manual de métodos de análises de solo. Rio de Janeiro: Centro Nacional de Pesquisa de Solos; 1997.

Fenner M, Thompson K. The ecology of seeds. Cambridge: Cambridge University Press; 2005.

Garwood NC. Tropical soil seed banks: a review. Ecology of Soil Seed Banks 1989; 149: 210.

Hanin N, Quaye M, Westberg E, Barazani O. Soil seed bank and among-years genetic diversity in arid populations of Eruca sativa Miller (Brassicaceae). Journal of Arid Environments 2013; 91: 151-154. http://dx.doi.org/10.1016/j. jaridenv.2013.01.004.

Hong J, Liu S, Shi G, Zhang Y. Soil seed bank techniques for restoring wetland vegetation diversity in Yeyahu Wetland, Beijing. Ecological Engineering 2012; 42: 192-202. http:// dx.doi.org/10.1016/j.ecoleng.2012.01.004.

Hosogi D, Kameyama A. Timing for the planting method using deciduous forest topsoil in surbuban Tokyo. Japanese Ecolological Engineering 2006; 26(2): 123-131. http:// dx.doi.org/10.1016/j.ecoleng.2005.06.014.

Iglesias-Fernández R, Carmen RGM, Matilla AJ. Progress in research on dry afterripening. Seed Science Research 2011; 21(1): 69-80. http://dx.doi.org/10.1017/ S096025851000036X.

Jha P, Norsworthy JK, Kumar V, Reichard N. Annual changes in temperature and light requirements for Ipomoea purpurea seed germination with after-ripening in the field following dispersal. Crop Protection 2015; 67(1): 84-90. http://dx.doi.org/10.1016/j.cropro.2014.09.021.

Jurand BS, Abella SR, Suazo AA. Soil seed bank longevity of the exotic annual grass Bromus rubens in the Mojave Desert, USA. Journal of Arid Environments 2013; 94(1): 68-75. http://dx.doi.org/10.1016/j.jaridenv.2013.03.006.

Kaeser M, Kirkman LK. Seed longevity of 12 native herbaceous species in a fire-maintained pine savanna after 8years of burial. Forest Ecology and Management 2012; 281: 68-74. http://dx.doi.org/10.1016/j.foreco.2012.06.019.

Kanazawa Y, Xia K, Tan HY, Suzuki K. Seed storage longevity of Hosta sieboldiana (Asparagaceae). South African Journal of Botany 2015; 98: 6-9. http://dx.doi. org/10.1016/j.sajb.2015.01.009.

Li YY, Dong SK, Wen L, Wang XX, Wu Y. Soil seed banks in degraded and revegetated grasslands in the alpine region of the Qinghai-Tibetan Plateau. Ecological Engineering 2012; 49: 77-83. http://dx.doi.org/10.1016/j. ecoleng.2012.08.022.

Limón Á, Peco B. Germination and emergence of annual species and burial depth: implications for restoration 
ecology. Acta Oecologica 2016; 71: 8-13. http://dx.doi. org/10.1016/j.actao.2016.01.001.

Long RL, Gorecki MJ, Renton M, Scott JK, Colville L, Goggin DE et al. The ecophysiology of seed persistence: a mechanistic view of the journey to germination or demise. Biological Reviews of the Cambridge Philosophical Society 2015; 90(1): 31-59. PMid:24618017. http://dx.doi. org/10.1111/brv.12095.

Long Y, Tan DY, Baskin CC, Baskin JM. Seed dormancy and germination characteristics of Astragalus arpilobus (Fabaceae, subfamily Papilionoideae), a central Asian desert annual ephemeral. South African Journal of Botany 2012; 83: 68-77. http://dx.doi.org/10.1016/j.sajb.2012.06.010.

Lorenzi H. Árvores brasileiras: manual de identificação e cultivo de plantas arbóreas do Brasil. Nova Odessa: Plantarum; 2002.

Mandák B, Placková I. How does population genetic diversity change over time? An experimental seed bank study of Atriplex tatarica (Chenopodiaceae). Flora 2009; 204(6): 423-433. http://dx.doi.org/10.1016/j.flora.2008.05.005.

Marchiori JNC, Alves FS. Nota sobre a distribuição geográfica de Peltophorum dubium (Spreng.) Taub. no Rio Grande do Sul. Balduinia 2014; 33(1): 27-31.

Mendoza-Hernandez PE, Orozco-Segovia A, Meave JA, Valverde T, Martínez-Ramos M. Vegetation recovery and plant facilitation in a human disturbed lava field in a megacity: searching tools for ecosystem restoration. Plant Ecology 2013; 214(1): 153-167. http://dx.doi.org/10.1007/ s11258-012-0153-y.

Mendoza-Hernández PE, Rosete-Rodríguez A, SánchezCoronado ME, Orozco S, Pedrero-López L, Méndez I et al. Vegetation patches improve the establishment of Salvia mexicana seedlings by modifying microclimatic conditions. International Journal of Biometeorology 2014; 58(5): 853-866. PMid:23605562. http://dx.doi.org/10.1007/ s00484-013-0665-8.

Motta MS, Davide AC, Ferreira RA. Longevidade de sementes de mutamba (Guazuma ulmifolia Lam.Sterculiaceae) no solo em condições naturais. Revista Brasileira de Sementes 2006; 28(2): 7-14. http://dx.doi. org/10.1590/S0101-31222006000200002.
Murdoch AJ, Ellis RH. Dormancy, viability and longevity. In: Fenner M, editor. Seeds: the ecology of regeneration in plant communities. 2nd ed. Wallingford: CAB International; 2000.

O’Donnell J, Fryirs KA, Leishman MR. Seed banks as a source of vegetation regeneration to support the recovery of degraded rivers: a comparison of river reaches of varying condition. The Science of the Total Environment 2016; 542(Pt A): 591-602. PMid:26544888. http://dx.doi. org/10.1016/j.scitotenv.2015.10.118.

Sánchez-Coronado ME, Márquez-Guzmán J, RosasMoreno J, Vidal-Gaona G, Villegas M, Espinosa-Matías $S$ et al. Mycoflora in exhumed seeds of Opuntia tomentosa and its possible role in seed germination. Applied and Environmental Soil Science 2011; 2011(1): 1-8. http:// dx.doi.org/10.1155/2011/107159.

Shen Y, Zhao C, Liu W. Seed vigor and plant competitiveness resulting from seeds of Eupatorium adenophorum in a persistent soil seed bank. Flora-Morphology, Distribution. Functional Ecology of Plants 2011; 206(11): 935-942. http:// dx.doi.org/10.1016/j.flora.2011.07.002.

Soriano D, Huante P, Gamboa-deBuen A, Orozco-Segovia A. Effects of burial and storage on germination and seed reserves of 18 tree species in a tropical deciduous forest in Mexico. Oecologia 2014; 174(1): 33-44. PMid:24002712. http://dx.doi.org/10.1007/s00442-013-2753-1.

Suriyong S, Krittigamas N, Pinmanee S, Punyalue A, Vearasilp S. Influence of storage conditions on change of hemp seed quality. Agriculture and Agricultural Science Procedia 2015; 5: 170-176. http://dx.doi.org/10.1016/j. aaspro.2015.08.026.

van Klinken $\mathrm{RD}$, White AJ. The role of pre-and postdispersal seed predation in determining total seed loss. Basic and Applied Ecology 2014; 15(7): 581-589. http:// dx.doi.org/10.1016/j.baae.2014.08.012.

Williams L, Reich P, Capon SJ, Raulings E. Soil seed banks of degraded riparian zones in southeastern Australia and their potential contribution to the understorey vegetation. River Research and Applications 2008; 24(7): 1002-1017. http://dx.doi.org/10.1002/rra.1123. 